Address for Correspondence: Dr. Gautam Rawal, Max Super Speciality Hospital Saket, New Delhi, India; Email: drgautamrawal@hotmail.com

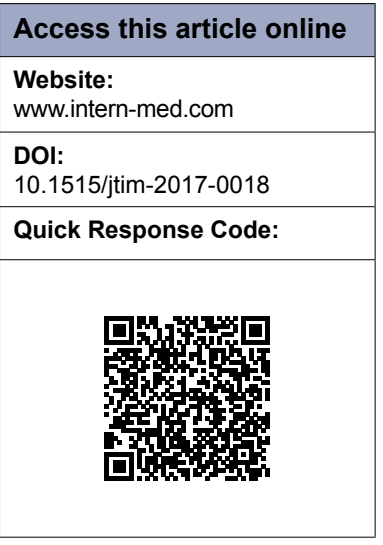

\title{
H1N1 influenza induced acute respiratory distress syndrome rescued by extracorporeal membrane oxygenation: A case report
}

\author{
Gautam Rawal $^{1}$, Raj Kumar ${ }^{1}$, Sankalp Yadav ${ }^{2}$, R Sujana ${ }^{3}$ \\ ${ }^{1}$ Respiratory Intensive Care, Max Super Speciality Hospital Saket, New Delhi, India; \\ 2Department of Medicine \& TB, Chest Clinic Moti Nagar, \\ North Delhi Municipal Corporation, New Delhi, India; \\ ${ }^{3}$ Infection Control Unit, Max Super Specialty Hospital, Saket, New Delhi, India
}

\section{ABSTRACT}

Extracorporeal Membrane Oxygenation (ECMO) is now a recognized revolutionary technology, which has emerged as a life-saving therapeutic option for patients with potentially reversible severe respiratory failure who fail to respond positively with the conventional ventilation therapy. Severe acute respiratory distress syndrome (ARDS) is associated with high morbidity and mortality, and ECMO has been proven to increase the survival rates in these patients by improving the gas exchange and thus preventing the further hypoxia and catecholamine induced multi-organ damage. The authors present a case of $\mathrm{H} 1 \mathrm{~N} 1$ influenza related severe ARDS who was successfully rescued by the early use of ECMO. The authors recommend the early use of ECMO to be incorporated in the management of severe refractory ARDS caused due to a potentially reversible cause.

Key words: acute respiratory distress syndrome, extracorporeal membrane oxygenation, H1N1 influenza, hypoxia, swine flu

\section{INTRODUCTION}

The H1N1 novel influenza A (swine flu) virus illness, which is associated with high morbidity and mortality, is known to occur as pandemic and epidemic worldwide. The infection causes severe hypoxemic respiratory failure or the acute respiratory distress syndrome (ARDS) with multi-organ involvement. ${ }^{[1,2]}$ The H1N1 pandemic of 2009 saw the emergence of veno-venous extra corporeal membrane oxygenation (VV- ECMO) as a rescue therapy in the cases of H1N1 swine flu related ARDS which were refractory to the conventional therapy ${ }^{[2]}$ The authors present a case of the successful management of an obese patient with H1N1 influenza pneumonia-induced severe ARDS using the VV-ECMO.

\section{CASE PRESENTATION}

A 44-year-old lady, who was a known case of systemic hypertension with type 2 diabetes mellitus, polycystic ovarian syndrome and obesity, presented in triage with complaints of sore throat, fever and dry cough present for three days and progressive shortness of breath for two days. On arrival in the emergency department, she was in a state of severe respiratory distress with severe hypoxia $\left(\mathrm{SPO}_{2} 54 \%\right.$ on room air) with peripheral cyanosis, tachypnea (respiratory rate $36 / \mathrm{min}$ ) and hypotension (BP 90/60 mmHg). Her arterial blood gas (ABG) showed hypoxemia with $\mathrm{PaCO}_{2}$ $56 \mathrm{mmHg}, \mathrm{PaCO}_{2} 55 \mathrm{mmHg}, \mathrm{pH} 7.30$ despite being on $100 \%$ oxygen with nonrebreather oxygen mask with oxygen@ $15 \mathrm{~L} / \mathrm{min}$. She was intubated and ventilated in view of persisting severe respiratory distress, hypoxemia and hypercarbia with shock. She was initiated on norepinephrine infusion, with an aim to keep the mean arterial blood pressure above $65 \mathrm{mmHg}$ and transferred to the respiratory intensive care unit (RICU) for further management. In the RICU, she remained hypoxemic 
despite the optimum ventilatory support using pressure controlled ventilation with high positive end expiratory pressure (PEEP) of $14 \mathrm{~cm}$ water, with plateau pressure $34 \mathrm{~cm}$ water and on $\mathrm{FiO}_{2}$ of 1.0 with her blood oxygen saturation $\left(\mathrm{SpO}_{2}\right)$ remaining around $78-80 \%$. Her ABG on the above ventilator settings showed poor oxygenation and ventilation with a $\mathrm{PaO}_{2} / \mathrm{FiO}_{2}$ ratio of $65, \mathrm{pH} 7.28$, and $\mathrm{PaCO}_{2} 60 \mathrm{mmHg}$, lung compliance of $20 \mathrm{~mL} / \mathrm{cm}$ $\mathrm{H}_{2} \mathrm{O}$ and chest $\mathrm{X}$-ray showed bilateral diffuse lung infiltrates suggestive of severe ARDS (Figure 1), with a Murray score of 3.5. Septic profile screening for pneumonia along with a nasopharyngeal swab for reverse transcriptase polymerase chain reaction (RT-PCR) H1N1 was sent. She was empirically started on broad-spectrum intravenous antibiotics (Piperacillin-Tazobactum, Clarithromycin), antiviral (Olsetamivir) in suspicion of H1N1 pneumonia and other supportive care. Her blood investigations showed mildly deranged renal functions with serum urea $70 \mathrm{mg} / \mathrm{dL}$ (normal range $7-20 \mathrm{mg} / \mathrm{dL}$ ) and serum creatinine $1.8 \mathrm{mg} / \mathrm{dL}$ (normal range $0.5-1.3 \mathrm{mg} / \mathrm{dL}$ ) with a normal leucocyte count and liver functions. Her serum procalcitonin level was also normal $(0.4 \mathrm{ng} / \mathrm{mL})$. Her electrocardiogram was normal on the first day of admission, but the next day there were electrocardiogram changes noticed ( $\mathrm{T}$ wave inversion leads V4-6 with no changes in the concomitant leads) with mildly elevated troponin I levels of $0.08 \mathrm{ng} / \mathrm{mL}$ (normal $<0.04 \mathrm{ng} / \mathrm{mL}$ ). Her echocardiogram on the day of admission was reported normal with left ventricular ejection fraction (LVEF) of 55\% and was repeated the next day, which showed global hypokinesia of the myocardium with no regional wall motion abnormality (RWMA) and decreased LVEF of $40-45 \%$. These changes were s/o stress induced cardiomyopathy. In the view of refractory hypoxemia, the option of prone position ventilation was considered, but abandoned due to increasing hemodynamic instability, severe abdominal obesity and lack of experience

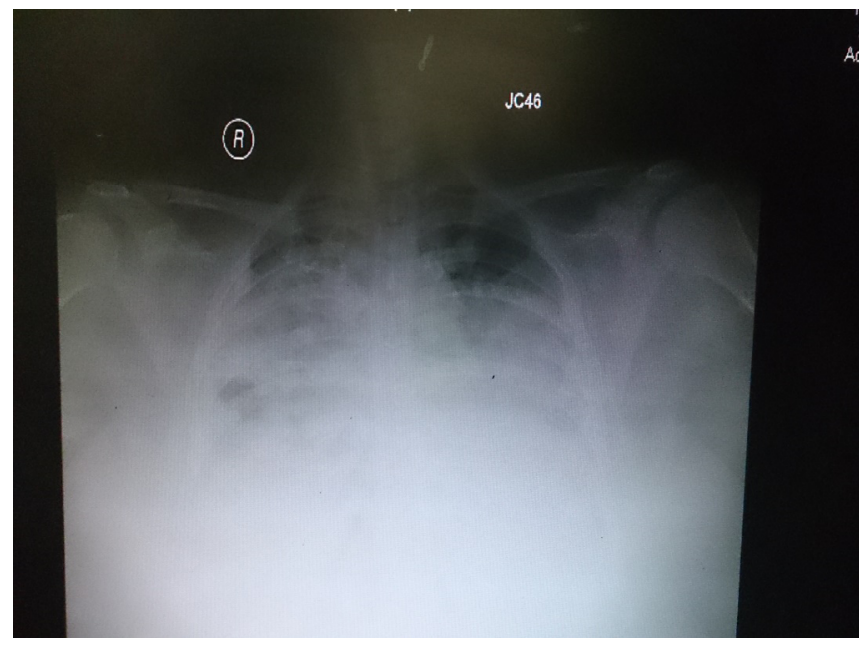

Figure 1: Chest X-ray showing bilateral extensive lung infiltrates s/o ARDS of the nursing staff. On the second day of admission, the possibility of ECMO support was discussed with attendants, to which they agreed. She was initiated on VV ECMO (Maquet system) with 24 F multistage Medtronic's drain cannulae in the right femoral vein and $21 \mathrm{~F}$ return cannulae in the right internal jugular vein (IJV) (Figure 2). The initial settings of ECMO being flow of $50 \mathrm{~mL} / \mathrm{kg} / \mathrm{min}$, speed 3200 rotations/min, fraction of delivered oxygen $\left(\mathrm{FdO}_{2}\right) 100 \%$ and the sweep of $8 \mathrm{~L} / \mathrm{min}$, her ventilator support reduced to resting lung ventilation (PEEP $10 \mathrm{~cm}$ $\mathrm{H}_{2} \mathrm{O}$ and pressure control above PEEP $20 \mathrm{~cm} \mathrm{H}_{2} \mathrm{O}$ ) and unfractionated heparin infusion to aim activated clotting time to 160-180 s. Her $\mathrm{SpO}_{2}$ immediately improved to 98\% on ECMO and the hemodynamic parameters started to stabilize with tapering of norepinephrine infusion. Bronchoscopy was done in view of bilateral pneumonic infiltrates, which revealed mild hemorrhagic secretions. Bronchial alveolar lavage cultures were sterile. Her throat swab for H1N1 RT-PCR was positive. She was maintained on a minimal sedation with fentanyl infusion to aim a Ramsay sedation score of 2-3. Patient showed gradual improvement in oxygenation (improved $\mathrm{PaO}_{2} / \mathrm{FiO}_{2}$ ratio) with increasing lung compliance (increased to $50-60 \mathrm{~mL} /$ $\mathrm{cm} \mathrm{H}_{2} \mathrm{O}$ ) on the ventilator over the next 72 hours. Her ECMO support was reduced (gradual decrease of $\mathrm{FdO}_{2}$ and sweep) over five days and was stopped on the fifth day of the ECMO initiation. The ECMO invasive cannulae were removed the next day and the patient was able to sustain well on the ventilator. She was gradually weaned off from the ventilator support in the following next three days and a tracheal extubation was done. She initially required oxygen support, which was also gradually tapered off. She showed good recovery and was transferred to room, where she remained stable and discharged in a stable condition. On the day of discharge her echocardiogram was repeated, which now was normal with LVEF 55\% with no RWMA

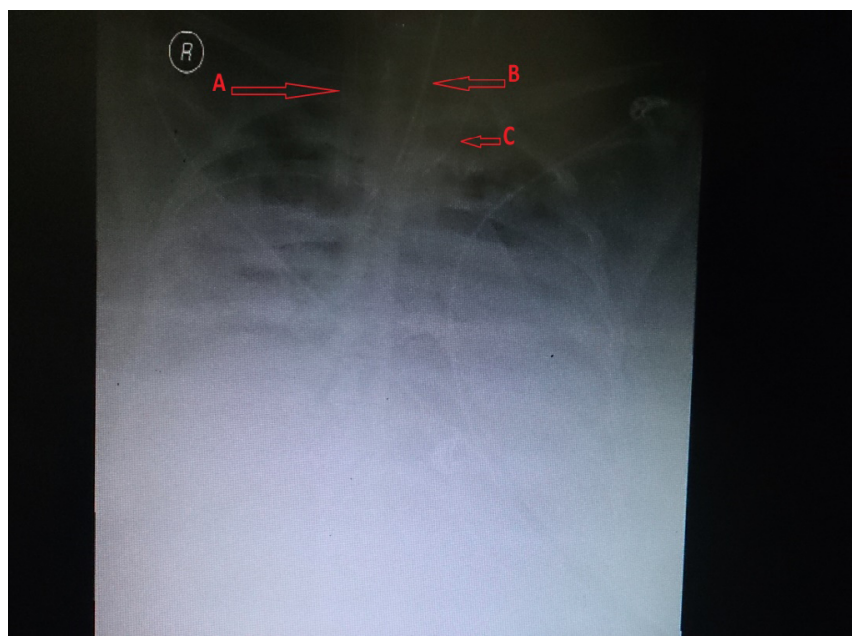

Figure 2: Chest X-ray showing: A-ECMO cannula in right IJV, B-endotracheal tube in situ, C-central venous line in left IJV 
and her troponin I level were also normal. She is being regularly followed-up as an outpatient and is doing well without any residual lung injury.

ECMO proved to be a life-saving management in our patient by supporting her and maintaining the blood oxygenation with decreased ventilator induced injury, until resolution of the H1N1 pneumonia.

\section{DISCUSSION}

The 2009 pandemic of swine flu made the clinicians aware of the lethal effects of the H1N1 virus infection, mainly due to the ARDS, with the ECMO therapy showing a real promising role to tackle this. ${ }^{[2,3]}$ ARDS is a life threatening hypoxemic respiratory failure of varied etiologies, carrying high morbidity and mortality. ${ }^{[4]}$ Severe ARDS is defined by the Berlin definition as $\mathrm{PaO}_{2} / \mathrm{FiO}_{2}$ ratio $\leq 100 \mathrm{mmHg}$ in a patient on a ventilator with a PEEP $\geq 5 \mathrm{~cm} \mathrm{H}_{2} \mathrm{O} \cdot{ }^{[4]}$ Limited therapeutic options have been proven to decrease mortality associated with severe ARDS, which include lowtidal volume mechanical ventilation or the lung protective ventilation (using tidal volume of $6 \mathrm{~mL} / \mathrm{kg}$ body weight), prone ventilation and ECMO support therapy in severe refractory cases not responding to the conventional treatment methods. ${ }^{[4,5]}$ ECMO has shown good results in saving lives of the patients in ARDS due a reversible cause, especially due to the increasing experience and expertise of the intensivists in using VV-ECMO. It should be understood that the ECMO itself is not a treatment modality; it actually prevents the lung from further injury by decreasing the incidence of ventilator-induced lung injury due to mechanical ventilation with high PEEP and prevents multi-organ failure due to hypoxemia and high vasopressor requirement. ${ }^{[5,6]}$ Thus, ECMO support provides time for the lungs to recover from the potentially reversible cause such as pneumonia. In the presented case, the patient may not have survived with the persistent hypoxia, hypercarbia, and increasing vasopressor requirement despite high optimum ventilatory support. ECMO support helped in preserving the end-organ failure and provided the time for the lungs to recover from severe H1N1 pneumonia.

ECMO is either veno-arterial (VA) ECMO (used in cardiac or cardiorespiratory failure) or veno-venous (VV) ECMO (used in respiratory failure with a reversible etiology and without cardiac compromise). ${ }^{[5]}$ The Extracorporeal Life Support Organization (ESLO) has recommended the use of ECMO in cases where the expected mortality, considering the Murray score, is more than $80 \%$, but can be considered early if the expected mortality is more than $50 \%{ }^{[5]}$ The VV-ECMO use not only needs expertise, but it is associated with complications like bleeding from various sites (major complication), deep vein thrombosis of the lower limb where the ECMO cannula is inserted, sepsis due to the large bore invasive intravenous lines, systemic thromboembolism neurologic complications (seizures, hemorrhage, infarcts), acute renal injury and hemolysis. ${ }^{[7]}$

Antiviral drugs form the mainstay of the clinical treatment of H1N1 influenza and are proven to be most effective if started within 48 hours of clinical symptoms; thus, they should be administered empirically in all cases of suspected H1N1 viral infection. ${ }^{[1]}$ The center for disease control (CDC) recommends the anti-viral Drugs-Oseltamivir or Zanamivir, to be given as early as possible even before the result of the diagnostic test is available. The negative results of the rapid antigen test (RT-PCR of nasopharyngeal secretions) do not exclude the possibility of H1N1 infection in severe cases of ARDS. ${ }^{[1,3,8]}$ The standard dose of Oseltamivir in adults is $75 \mathrm{mg}$ orally, twice daily for five days. ${ }^{[1]}$ Concomitant broad spectrum antibiotics are also recommended as the patients with the viral infection have a high predilection for secondary bacterial infection. ${ }^{[1]}$ The supportive treatment includes good nutrition to the patient during the critical illness, ${ }^{[0]}$ regular counseling of the awake patient and also the relatives (as they are susceptible to develop post-traumatic stress disorder after recovering from the critical illness), and regular physical therapy. ${ }^{[10]}$

\section{CONCLUSIONS}

The H1N1 influenza pneumonia is associated with high morbidity and mortality due to its rapid progression to ARDS. The ECMO technology has seen vast improvement in the past decade and with the experience growing in its use and the promising results. It is recommended that all the patients with severe respiratory failure or ARDS and refractory to the conventional management should be considered for ECMO (or referred to a center with ECMO facility) early in the course of management. ECMO is not a treatment modality, but by its virtue of stabilizing the gas exchange and consequently the hemodynamics of the patient, it prevents the ongoing hypoxic organ damage and provides time for the lungs to recover, thereby increasing the survival rates in these critical patients with high mortality rates.

\section{Conflict of Interest}

None declared.

\section{REFERENCES}

1. Rewar S, Mirdha D, Rewar P. Treatment and Prevention of Pandemic H1N1 Influenza. Ann Glob Health 2015;81:645-53.

2. Peek GJ, Mugford M, Tiruvoipati R, Wilson A, Allen E, Thalananyet $\mathrm{MM}$, et al. Efficacy and economic assessment of conventional ventilatory support versus extracorporeal membrane oxygenation for severe adult 
respiratory failure (CESAR): a multicentre randomised controlled trial. Lancet 2009;374:1351-63.

3. Louie JK, Acosta M, Winter K, Jean C, Gavali S, Schechter R, et al. Factors associated with death or hospitalization due to pandemic 2009 influenza A (H1N1) infection in California. JAMA 2009;302:1896902.

4. Rawal G, Yadav S, Kumar R. Acute respiratory distress syndrome: An update and review. J Transl Intern Med. ISSN (Online) 2224-4018, DOI: 10.1515/jtim-2016-0012, May 2016.

5. Rawal G, Kumar R, Yadav S. ECMO Rescue Therapy in Diffuse Alveolar Haemorrhage: A Case Report with Review of Literature. J Clin Diagn Res 2016;10:OD10-11.

6. Anand S, Jayakumar D, Aronow WS, Chandy D. Role of extracorporeal membrane oxygenation in adult respiratory failure: an overview. HospPract (1995) 2016;44:76-85.
7. Makdisi G, Wang IW. Extra corporeal membrane oxygenation (ECMO) review of a life saving technology. J Thorac Dis 2015;7:E166-76.

8. Tabarsi P, Moradi A, Marjani M, Baghaei P, Hashemian SM, Nadji SA, et al. Factors associated with death or intensive care unit admission due to pandemic 2009 influenza A (H1N1) infection. Ann Thorac Med 2011;6:91-5.

9. Rawal G, Yadav S. Nutrition in chronic obstructive pulmonary disease: A review. J Transl Intern Med 2015;3:151-4.

10. Rawal G, Yadav S, Kumar R. Post-Traumatic Stress Disorder: A Review from Clinical Perspective. Int J Indian Psychol 2016;3:156-64.

How to cite this article: Rawal G, Kumar R, Yadav S, Sujana R. H1N1 influenza induced acute respiratory distress syndrome rescued by extracorporeal membrane oxygenation: A case report. J Transl Intern Med 2017; 5: 182-85. 